\title{
CD4+CD25-Foxp3+ T cells as a marker of disease activity and organ damage in systemic lupus erythematosus patients
}

Nermine El-Maraghy ${ }^{1}$, Mona S. Ghaly ${ }^{2}$, Omar Dessouki ${ }^{3}$, Samah Ismail Nasef ${ }^{2}$, Lobna Metwally ${ }^{1}$

\author{
1Department of Microbiology and Immunology, Faculty of Medicine, Suez Canal \\ University, Ismailia, Egypt \\ ${ }^{2}$ Department of Rheumatology and Rehabilitation, Faculty of Medicine, Suez Canal \\ University, Ismailia, Egypt \\ ${ }^{3}$ Department of Clinical Pathology, Faculty of Medicine, Suez Canal University, \\ Ismailia, Egypt
}

Submitted: 26 May 2016

Accepted: 5 August 2016

Arch Med Sci 2018; 14, 5: 1033-1040

DOI: https://doi.org/10.5114/aoms.2016.63597

Copyright $\odot 2016$ Termedia \& Banach

\section{Abstract}

Introduction: T regulatory cells (Treg) play an important role in the maintenance of immune cell homeostasis, as it has been reported that CD4+CD25+ T cells suppress the auto-reactive responses in autoimmune diseases such as systemic lupus erythematosus (SLE). The clinical significance of the recently identified population of CD4+CD25-Foxp3+ T cells and whether they are associated with particular organ involvement is still not clear. So, the aim of our study was to evaluate the presence of CD4+CD25-Foxp3+ cells in SLE patients in comparison to healthy controls and to determine whether their frequency is associated with disease activity and particular clinical manifestations in these SLE patients.

Material and methods: The frequency of CD4+CD25-Foxp3+ T cells was analyzed in 56 female SLE patients and 30 healthy female control subjects, using flow cytometry (FACS). CD4+CD25-Foxp3+ T cells were correlated with clinical and laboratory data and the SLE Disease Activity Index (SLEDAI).

Results: The level of CD4+CD25-Foxp3+ T cells was significantly increased in SLE patients $(15.57 \pm 4.32 \%)$ as compared with the control group (2.46 $\pm 0.65 \%)$. A significant correlation was observed for the percentage of CD4+CD25-Foxp3+ $T$ cells with clinical disease activity scores and disease duration ( $r=0.6, p<0.001 ; r=0.3, p=0.02$ respectively). It was also positively correlated with renal impairment and hematological involvement.

Conclusions: Systemic lupus erythematosus patients exhibited an altered level of their CD4+Foxp3+ T cells with increased levels of CD4+CD25-Foxp3+ cells.

Key words: T regulatory cells, systemic lupus erythematosus, organ involvement, renal impairment.

\section{Introduction}

T regulatory cells (Treg) play an important role in the maintenance of immune cell homeostasis. Specifically, Treg maintain regulation in the immune system through dominant negative regulation of other immune cells [1]. They are classified into natural and adaptive cells: The natural Treg are CD4+CD25+ T cells [2]. Interleukin 2 (IL-2) and transforming growth factor $\beta$ (TGF- $\beta$ ) help naïve CD4+ T cells to differentiate into

\author{
Corresponding author: \\ Samah Ismail Nasef \\ Rheumatology and \\ Rehabilitation Department \\ Faculty of Medicine \\ Suez Canal University \\ 41111 Ismailia, Egypt \\ Phone: +201093155272 \\ Fax: +20643356539 \\ E-mail:dr.samah.ismaeel@ \\ gmail.com
}


Foxp3+ Treg cells in the periphery [3, 4]. Although the temporary up-regulation of Foxp3 expression has been observed in human $T$ cells upon its activation, still conflicting data have been reported concerning the suppressive ability of $T$ cells with the transient expression of Foxp3 [5-7].

Though Foxp3 expression is primarily restricted to the CD4+CD25+ cell population, it can be expressed at a low level in CD4+CD25- cells [8, 9]. Bonelli and his colleagues found that the proportion of CD4+CD25-Foxp3+ T cells within CD4+ lymphocytes was nearly $8 \%$ [10].

Systemic lupus erythematosus (SLE) is a disease characterized by altered immunity with hyperactive $T$ and $B$ cells. Also, it is very apparent that Treg cells are defective in SLE [11] and Foxp3 numbers appeared to be decreased during the course of the disease [12]. These finding suggested that there is a link between the clinical activity of SLE and the number and function of Treg cells.

As a reduction in Treg numbers and Treg deficiencies have been reported in several studies conducted in mouse models and in SLE patients [13-15], they suffer from deficient Treg-mediated suppression $[11,12]$, and it may lead to undisturbed suppressive activity of the Treg cells [16]. Likewise, it has been reported that in SLE, there is either a reduction in the number of Treg cells [16-18], no change in number [11], or even a rise in number [19].

It has been reported that there is a novel subset of CD4+Foxp3+ that does not express CD25 [10] and suppresses the T-cell proliferation in vitro. It was observed that SLE patients have a high level of this CD4+CD25-Foxp3+ subset [20-24] as they produce a high level of IL-2 and other cytokines than CD25 $5^{\text {high }}$ Foxp3+ subsets do. So, we aimed to evaluate the frequency of CD4+CD25- Foxp3+ cells in SLE in comparison to healthy control subjects and its association with clinical manifestations in SLE patients.

\section{Material and methods}

The present study included 56 SLE female patients with a mean age $27.8 \pm 6.4$ years who fulfilled the American College of Rheumatology (ACR) classification criteria for SLE [25]. They were recruited from the Rheumatology Department, Suez Canal University Hospital, Ismailia, Egypt. The control group comprised 30 healthy women with a mean age of $29.3 \pm 5.8$ years. This study was approved by the ethical committee of Suez Canal University. Informed consent was obtained from all participating subjects. Both patient and control groups were from the Suez Canal area.

Clinical manifestations were defined as either present or absent based on the definitions of the SLE classification's criteria at the time of blood sample collection from the patients. The following items were considered to be indicative for organ involvement: active joint defined as arthritis with swelling; active skin lesions defined as lupus rash, photosensitivity or discoid lupus; active hematologic involvement defined as leucopenia, lymphopenia, thrombocytopenia or hemolytic anemia; active renal involvement defined as urinary casts and/or proteinuria $>0.5 \mathrm{gm}$ protein $/ 24 \mathrm{~h}$, and active central nervous system (CNS) defined as lupus headache, psychosis or seizures.

Disease activity was assessed using the Systemic Lupus Erythematosus Disease Activity Index (SLEDAI). Clinical manifestations of SLE in the patient group included CNS manifestations (seizure, psychosis, visual disturbances, cranial nerve disorder, and lupus headache), vascular manifestations (vasculitis, Raynaud's phenomenon, and thromboembolism) and musculoskeletal manifestations (myositis and arthritis). The renal manifestations encompassed urinary casts, hematuria, proteinuria and pyuria. Rash, alopecia and mucosal ulcers were used to assess dermal clinical findings. Hematologic findings encompassed thrombocytopenia and leucopenia [26], while constitutional fever is defined as fever $\left(>38^{\circ} \mathrm{C}\right)$. A SLEDAl score $1-5$ was defined as mild disease activity, SLEDAI score 6-10 as moderate disease activity and a SLEDAI score of $>10$ as severe disease activity.

Laboratory work included autoantibodies (antinuclear antibodies (ANA), double stranded deoxyribonucleic acid (dsDNA), anti Ro and anti La). An indirect immunofluorescent assay, for semi-quantitative determination of anti-nuclear IgG antibodies (ANA) in patients' serum, NOVA LITE IFA HEp-2 ANA Complete Kit, was used. Double stranded DNA was detected by the indirect immunofluorescent assay for semi-quantitative determination of (dsDNA) in patient's serum (NOVA Diagnostics, Inc. San Diego, CA 92131 U.S.A.).

Antibodies to Ro/SSA and La/SSB were detected by solid-phase immunoassays. Human AntiSS-A (60/52 kDa) IgG ELISA is an indirect solid phase enzyme-linked immunosorbent assay (ELISA). It is designed for the quantitative measurement of IgG class autoantibodies directed against extractable nuclear antigens (Alpha Diagnostic Intl Inc., 6203 Woodlake Center Dr, San Antonio, USA).

\section{Treg subset study by flow cytometry}

Whole blood was collected in EDTA vacutainers tubes. Treg subsets regarding their CD25 and Foxp3 expression were identified by using monoclonal antibodies for the following markers: antiCD4 antibodies labeled by FITC fluorochrome, anti-FOXP3 labeled by PE fluorochrome and antiCD25 labeled by APC fluorochrome. Surface staining was done first, for both CD4 and CD25 following the manufacturer's instructions. Intra- 
cellular staining for FOXP3 using the FOXP3 BD kit followed the surface staining. Appropriate isotype controls were used. After staining data acquisition by FACS Calibur using Cell Quest software (BD Bio-sciences, San José, CA USA) and analysis by FlowJo software v.7.1.2 (Tree star) was performed. Lymphocytes were gated according to their light scatter properties and CD45 expression. CD4+ cells were gated. Treg subtypes were as follows: CD4+CD25+Foxp3-, CD4+CD25+FOXP3+ and CD4+CD25-FOXP3+.

\section{Statistical analysis}

Values are shown as number and percentage as well as mean and standard deviation (SD), unless otherwise specified. Lymphocyte subpopulations' proportions were compared using the Student $t$-test and the multiple comparison test one-way ANOVA. A $p$-value equal to or less than 0.05 was considered significant in all statistical tests. Statistical analyses and data plotting were performed using Microsoft Excel by Microsoft Inc. and SPSS (SPSS 22.0 by SPSS software Inc.).

\section{Results}

To determine whether CD4+CD25-Foxp3+ T cells' appearance is related to disease activity, patients were divided into 3 groups according to their disease activity scores. Demographic and clinical characteristics of SLE patients are presented in Tables I and II. Peripheral blood from the control group and patients with SLE was analyzed by fluorescence-activated cell sorting (FAC) for proportions of Treg subsets CD4+CD25+Foxp3+, CD4+CD25-Foxp3+, and CD4+CD25+Foxp3- in patients with SLE and control subjects (Figure 1 and Table III). CD4+CD25Foxp3+ $T$ cells were significantly increased in patients with SLE $(15.57 \pm 4.32 \%)$ as compared with the control group $(2.46 \pm 0.65 \%)$.

Comparing proportions of lymphocyte subpopulations using the ANOVA test showed a significant correlation between CD4+CD25-Foxp3+ T cells and disease activity $(p=0.018)$ in comparison to other Treg subsets.

When compared with the control group, significantly higher proportions of CD4+CD25-Foxp3+ $T$ cells were observed in cells from mild SLE patients $(6.1 \pm 0.7 \%)$, moderate active SLE patients $(10.5 \pm 1.4 \%)$ and more pronounced from severely active SLE patients (14.9 $\pm 2.3 \%)$. In agreement with this finding, a significant correlation was observed for the percentage of CD4+CD25-Foxp3+ $T$ cells with clinical disease activity scores and disease duration $(r=0.6, p<0.001 ; r=0.3, p=$ 0.02 respectively) (Figure $2 \mathrm{~A}$ ). No significant correlation was found between CD4+CD25-Foxp3+ T cells and age.
Association of CD4+CD25-Foxp3+ with clinical manifestations and production of antibodies in patients with SLE is shown in Table IV and Figures 2 B-E. A significant correlation was observed for the percentage of CD4+CD25-Foxp3+ T cells with urinary casts and proteinuria. In addition, we observed a significant correlation with anti-dsDNA antibody levels and a significant negative correlation between the levels of platelets and white blood cells and proportions of CD4+CD25-Foxp3+ $T$ cells. Other organ involvement showed no differences in proportions of CD4+CD25-Foxp3+ T cells.

Regarding the proportion of CD4+CD25-Foxp3+ in SLE patients with organ involvement, we observed a significant increase in proportions of CD4+CD25+Foxp3+ $T$ cells in patients with renal involvement presented by proteinuria (9.7 $\pm 2.1 \%)$ or urinary cast $(6.9 \pm 1.6 \%)$ as compared to patients with no renal involvement $(2.9 \pm 1.2 \%)$. Moreover, we observed increased proportions of

Table I. Demographic and clinical characteristics of systemic lupus erythematosus patients

\begin{tabular}{|c|c|}
\hline Characteristic & Value \\
\hline Gender, female, $n(\%)$ & $56(100)$ \\
\hline Disease duration, mean \pm SD [years] & $4.4 \pm 3.8$ \\
\hline ANA, positive, $n(\%)$ & $56(100)$ \\
\hline Anti-ds DNA Ab, positive, $n(\%)$ & $29(51.7)$ \\
\hline Anti-Ro, positive, $n(\%)$ & $13(23.2)$ \\
\hline Anti-La, positive, $n(\%)$ & $10(17.9)$ \\
\hline SLEDAI, mean \pm SD & $12.5 \pm 9.28$ \\
\hline \multicolumn{2}{|l|}{ Organ involvement/active, $n(\%)$ : } \\
\hline Arthritis & $31(55.4)$ \\
\hline Urinary casts & $12(21.4)$ \\
\hline Psychosis & $2(3.6)$ \\
\hline Lupus headache & $6(10.7)$ \\
\hline Skin involvement & $27(48.2)$ \\
\hline Proteinuria & $16(28.6)$ \\
\hline Leucopenia & $18(32.1)$ \\
\hline Thrombocytopenia & $12(21.4)$ \\
\hline Hemolytic anemia & 0 \\
\hline
\end{tabular}

Table II. SLEDAI score among SLE patients

\begin{tabular}{|lc|}
\hline Disease Activity Score & $\boldsymbol{N}(\%)$ \\
\hline Mild & $16(28.6)$ \\
\hline Moderate & $28(50)$ \\
\hline Severe & $12(21.4)$ \\
\hline
\end{tabular}



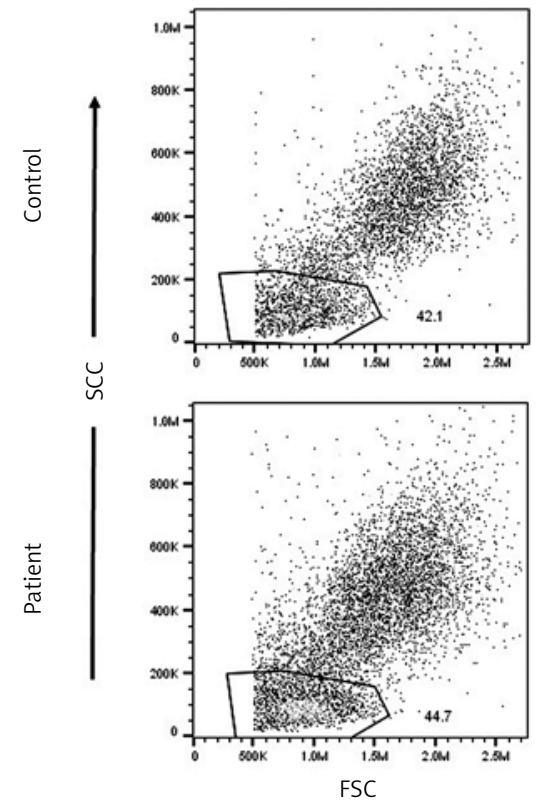
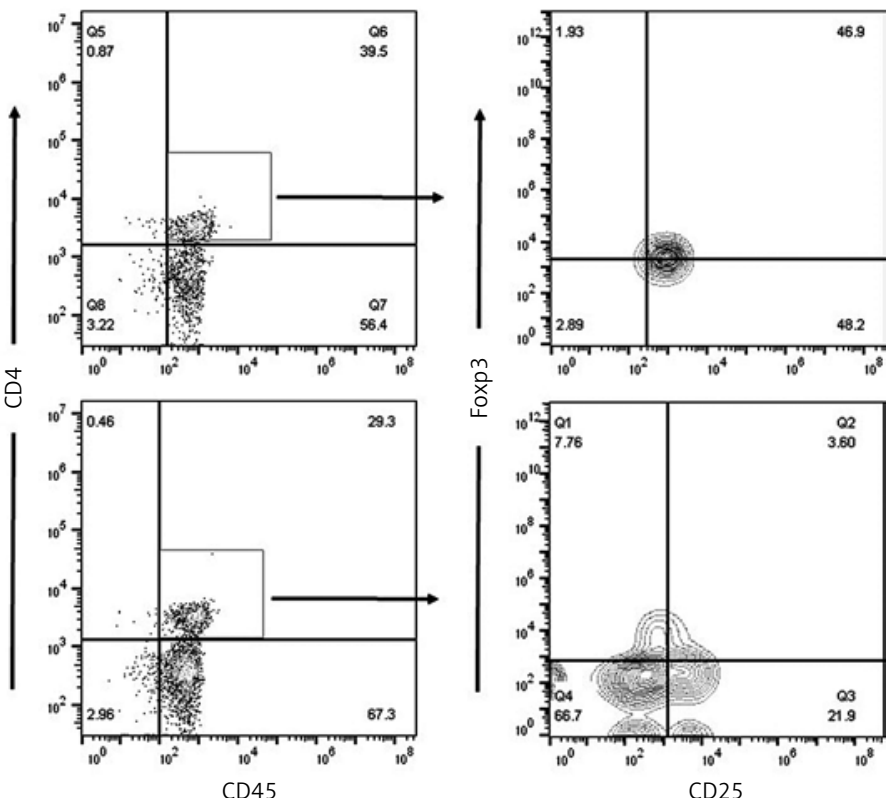

Figure 1. Acquisition of RBCs lysed PB and gating of lymphocytes on side-scattered light (SSC) versus forward-scattered light (FSC). Gated events were plotted against CD45 versus CD4. CD4+CD45+ events were gated, and plotted against CD25 versus Foxp3

Table III. CD4 subtypes among case and control groups

\begin{tabular}{|lccc|}
\hline CD4 subtype\% & SLE patients & Control & $P$-value \\
\hline CD4+ & $29.61 \pm 5.852$ & $32.53 \pm 5.511$ & 0.120 \\
\hline CD4+CD25+Foxp3+ & $3.100 \pm 1.4181$ & $35.24 \pm 4.794$ & $<.001$ \\
\hline CD4+CD25-Foxp3+ & $15.57 \pm 4.32$ & $2.46 \pm 0.65$ & $<.001$ \\
\hline CD4+CD25+Foxp3- & $13.25 \pm 6.780$ & $19.21 \pm 4.104$ & $<.001$ \\
\hline
\end{tabular}

CD4+CD25+Foxp3+ T cells in patients with hematologic involvement (either thrombocytopenia or leucopenia) (8.9 $\pm 1.9 \%)$ as compared to patients with no hematologic involvement (3.6 $\pm 0.7 \%)$.

\section{Discussion}

Tregs are essential controllers of immune tolerance. Substantial trials have been performed to outline the involvement of Tregs in the development of SLE[27]. They have suggested that quantitative and/or qualitative deficiencies of Treg are an important contributor to SLE development [28, 29].

Several researchers have investigated Foxp3-expressing CD4+ T cells in patients with SLE, revealing higher levels of CD4+CD25-Foxp3+ and lower levels of CD4+CD25+Foxp3+ T cells in SLE patients compared to normal controls [30-32].

According to these results, SLE patients may have faulty homeostatic control of different subsets of Foxp3+ cells. Our results revealed a significant decrease of the proportion of CD4+CD25+ Foxp3 in SLE compared to normal controls. These results were in accordance with other researchers who proved that SLE has a significantly decreased percentage and number of CD4+CD25 high Foxp3+ Tregs (corresponding to activated Tregs). They explained this depletion by the hypersensitivity of CD4+CD25+Foxp3+ Tregs to Fas-induced apoptosis. However, they failed to clarify the exact mechanisms responsible for this aggravated vulnerability to apoptosis [30, 32].

Thereafter, Long and Buckner [27] discussed this decrease as a global reduction in Treg, not preferential accumulation of Treg in lymph nodes, and this decrease in Treg number tends to be unique to SLE.

Moreover, researchers described the CD4+CD25T-cell subset to be relatively exceptional to SLE. Bonelli et al. [10] compared the CD4+CD25-Foxp3+ cells in different rheumatic diseases and found that it is only increased in SLE patients. Other researchers found that the levels of CD4+CD25-Foxp3+ correlated highly with the clinical disease activity but were not related to the patient's age, which was in contrast to murine studies where healthy animals showed an age-dependent increase of CD4+CD25-Foxp3+Treg [33-35].

However, up till now the origin and function of this subset have mostly been an issue of dis- 
A

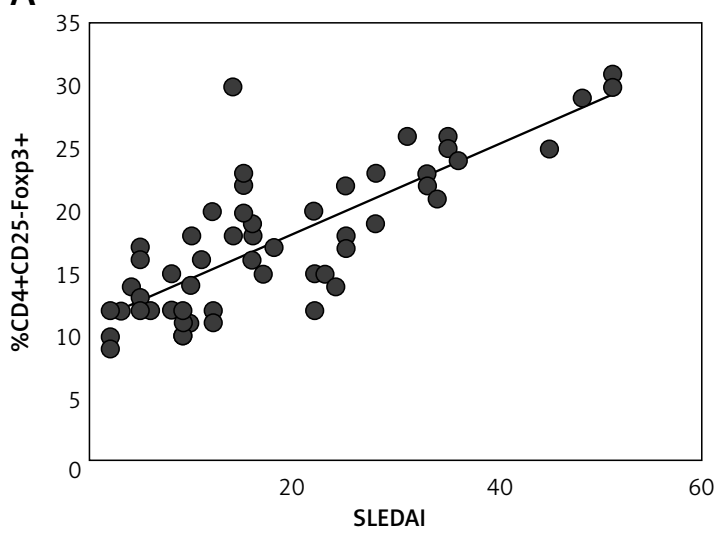

C

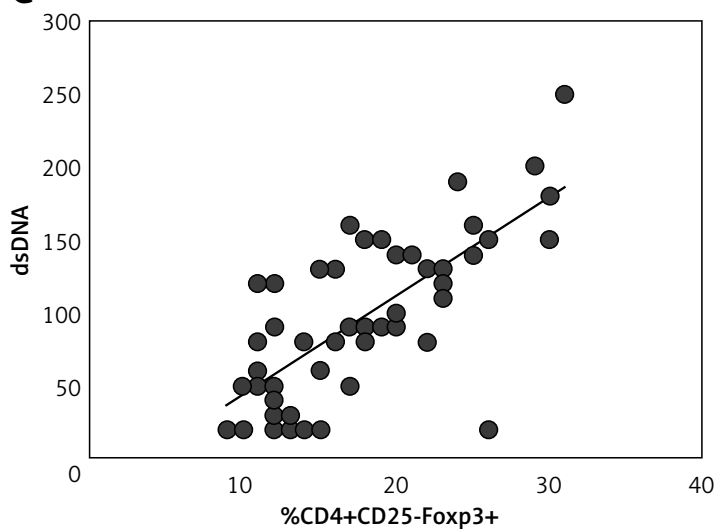

E

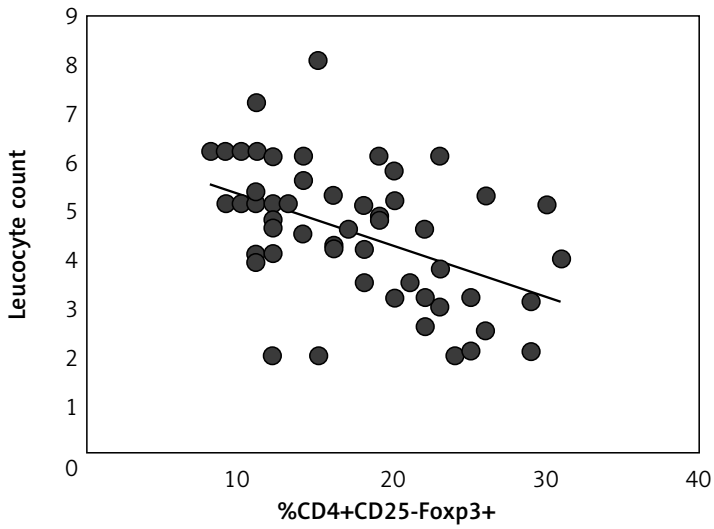

B

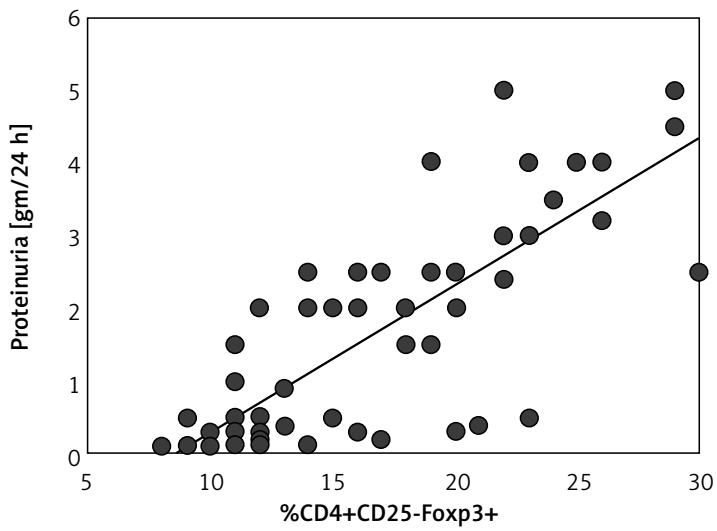

D

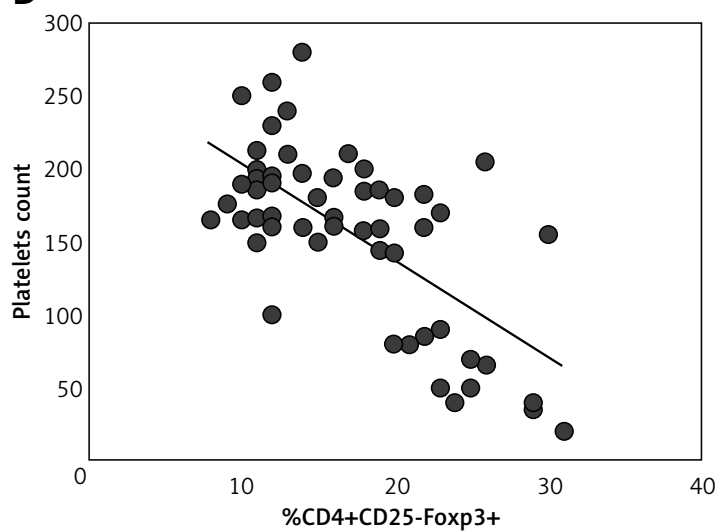

Figure 2. CD4+CD25-Foxp3+ $T$ cells correlate with SLE disease activity and organ involvement. A significant correlation was observed for the percentage of CD4+CD25-Foxp3+ T-cells and the SLE Disease Activity Index (SLEDAI) (A), proteinuria (B), levels of dsDNA antibodies (C), platelets (D) and leucocyte count (E)

cussion due to many causes. At first, activation may transiently induce their Foxp3 protein which may display non-Treg activity. Additionally, differentiation of naive CD4+ T cells into CD25+Foxp3+ and CD25-Foxp3+ Tregs, or so-called adaptive Tregs, can be induced by systemic inflammation. Lastly, the increased frequency of CD25-Foxp3+ $T$ cells in patients may be a compensation for the decreased CD25highFoxp3+ Tregs in active SLE, which may not be enough to control the autoimmune response [36].

Nevertheless, several studies have suggested that dendritic cells (DCs) may be accountable for this dysregulation [37, 38]. Interferon (IFN)- $\alpha$-pro-

ducing antigen-presenting cells as DCs may block Treg cell-mediated suppression function in SLE patients. Moreover, it was observed that the increased production by DCs of IL- 6 in lupus-prone mice can facilitate the reduced Treg function [38].

Lately, Bonelli et al. showed that CD4+CD25Foxp3+ cells phenotypically resemble CD4+ CD25+Foxp3+ Tregs rather than activated T cells. Furthermore, in vitro functional analysis revealed that they are able to suppress T-cell proliferation but not IFN- $\alpha$ production [10, 39]. Also, Yang et al., who worked on untreated new onset SLE, reported the increased percentage of this subset and suggested that these cells are Treg. They also reported 
Table IV. The association of CD4+CD25-Foxp3+ with clinical manifestations and production of antibodies in patients with SLE

\begin{tabular}{|lc|}
\hline Characteristic & $P$-value \\
\hline Arthritis & 0.978 \\
\hline Urinary casts & $0.001^{*}$ \\
\hline Psychosis & 0.657 \\
\hline Lupus headache & 0.254 \\
\hline Skin involvement & 0.421 \\
\hline \begin{tabular}{ll} 
Laboratory: \\
\hline Proteinuria
\end{tabular} & $0.000^{\star}$ \\
\hline Thrombocytopenia & $0.028^{*}$ \\
\hline Leukopenia & $0.016^{*}$ \\
\hline Anti-dsDNA & $0.019^{*}$ \\
\hline Anti-Ro & 0.87 \\
\hline Anti-La & 0.43 \\
\hline
\end{tabular}

*Statistically significant $(p \leq 0.005)$.

that there is a correlation between these cells and the titer of the dsDNA antibodies and that after treatment these cells decrease.

Our work agrees with the work of Yang et al. [22] and Bonelli et al. [10], as we analyzed the level of CD4+CD25-Foxp3+ T cells in SLE patients, and it was increased in SLE patients compared to the control group. Several studies have reported an increased proportion of Treg cells in SLE patients in relation to disease activity [40-42]. However, other studies showed an inverse correlation between the levels of CD4+CD25-Foxp3+ T cells and clinical disease activity [17, 43, 44]. This difference between our results and those reported by other investigators might be explained by the difference in the criteria used for diseases activity categorization of the patients, differences in the tissue of origin of the sample analyzed, and differences in gating strategy. Moreover, we found that there was a significant correlation with CD4+CD25Foxp3+ T cells and the anti-dsDNA antibody levels in patients' sera. The results of Bonelli et al. [39] were in accordance with our data, as they reported a significant correlation between CD4+CD25Foxp3+ $T$ cells and levels of anti-dsDNA antibodies, as well as Zhang et al. [20], who reported a direct correlation between the frequency of CD4+CD25-Foxp3+ $T$ cells and the concentration of antibodies against dsDNA. Suen et al. [24] described the increased proportions of CD25-Treg in patients with active SLE and with low complement levels. On the other hand, Lin et al. [42] did not find any correlation between CD4+CD25-Foxp3+ T cells and concentrations of anti-dsDNA antibodies in SLE patients. This disagreement may be due to the patients' criteria and treatment regime they are following.

Regarding organ involvement, most patients display multi-organ involvement as well as exacerbation and remission, with clinical manifestations as organ involvement can occur alone or in combination.

It was very clear from our work that the most affected systems are the hematological system and the renal system, which were very prominently affected, with the occurrence of proteinuria and renal casts with increased frequency of the CD4+CD25-Foxp3+ subset by $9.7 \pm 2.1 \%$ and 6.9 $\pm 1.6 \%$, respectively. There are few studies about the relation between organ involvement and different T-cell subsets. Bonelli et al. found that patients suffering from renal involvement, especially active nephritis, displayed increased proportions of CD4+CD25-Foxp3+ T cells [39]. They also showed a positive correlation between the extent of proteinuria and CD4+CD25-Foxp3, indicating the disease activity in active nephritis. This relation suggested the possibility of using the CD4+CD25-Foxp3+ subset as a biomarker to diagnose and monitor SLE patients with active renal involvement.

In addition, for those who suffer from active hematological manifestations including thrombocytopenia and leucopenia, it was determined in our study that the frequency of CD4+CD25-Foxp3+ $T$ cells was high in those patients. Bonelli et al. [39] also observed an increased level of these cells in SLE patients with active blood involvement, and this might be explained by the overlap of organ manifestation, as most SLE patients suffer from hematologic manifestations as well as renal involvement. So when they compared the level of CD4+CD25-Foxp3+ T cells in renal involvement only and that in combination with hematological manifestations, it was apparent that the frequency of these cells has a significant difference only in those with renal involvement.

It is obvious now that CD4+CD25-Foxp3+ T cell frequency correlates with disease activity, the level of dsDNA antibodies and complement levels and that the discrepancies between studies which confirmed these results and those that did not were due to the patients selected being active or inactive SLE patients and on different treatment regimens.

In conclusion, the results of our study confirmed that CD4+CD25-Foxp3+ T cells are increased in SLE patients with high disease activity and suggest that CD4+CD25-Foxp3+ T cells could be a suitable marker for monitoring SLE patients with lupus nephritis.

\section{Conflict of interest}

The authors declare no conflict of interest. 


\section{References}

1. Curotto de Lafaille MA, Lafaille JJ. Natural and adaptive foxp3+ regulatory T cells: more of the same or a division of labor? Immunity 2009; 30: 626-35.

2. Baecher-Allan C, Brown JA, Freeman GJ, et al. CD4+ CD25 high regulatory cells in human peripheral blood. J Immunol 2001; 167: 1245-53.

3. Zheng SG, Wang J, Wang P, et al. IL-2 is essential for TGF-beta to convert naive CD4+CD25- cells to CD25+ Foxp3+ regulatory $T$ cells and for expansion of these cells. J Immunol 2007; 178: 2018-27.

4. Davidson TS, DiPaolo RJ, Andersson J, et al. Cutting edge: IL-2 is essential for TGF-beta-mediated induction of Foxp3+ T regulatory cells. J Immunol 2007; 178: 4022-6.

5. Walker MR, Kasprowicz DJ, Gersuk VH, et al. Induction of FoxP3 and acquisition of T regulatory activity by stimulated human CD4+CD25- T cells. J Clin Invest 2003; 112: 1437-43.

6. Pillai V, Ortega SB, Wang CK, et al. Transient regulatory T-cells: a state attained by all activated human T-cells. Clin Immunol 2007; 123: 18-29.

7. Wang J, Ioan-Facsinay A, van der Voort El, et al. Transient expression of FOXP3 in human activated nonregulatory CD4+ T cells. Eur J Immunol 2007; 37: 129-38.

8. Fontenot JD, Rasmussen JP, Williams LM, et al. Regulatory $T$ cell lineage specification by the forkhead transcription factor foxp3. Immunity 2005; 22: 329-41.

9. Roncador G, Brown PJ, Maestre L, et al. Analysis of FOXP3 protein expression in human CD4+CD25+ regulatory T cells at the single-cell level. Eur J Immunol 2005; 35: 1681-91.

10. Bonelli M, Savitskaya A, Steiner CW, et al. Phenotypic and functional analysis of CD4+CD25-Foxp3+ $T$ cells in patients with systemic lupus erythematosus. J Immunol 2009; 182: 1689-95.

11. Miyara M, Amoura Z, Parizot C, et al. Global natural regulatory $T$ cell depletion in active systemic lupus erythematosus. J Immunol 2005; 175: 8392-400.

12. Wu HY, Staines NA. A deficiency of CD4+CD25+ T cells permits the development of spontaneous lupus-like disease in mice, and can be reversed by induction of mucosal tolerance to histone peptide autoantigen. Lupus 2004; 13: 192-200.

13. Monk CR, Spachidou M, Rovis F, et al. MRL/Mp CD4+,CD25- T cells show reduced sensitivity to suppression by CD4+,CD25+ regulatory T cells in vitro: a novel defect of $T$ cell regulation in systemic lupus erythematosus. Arthritis Rheum 2005; 52: 1180-4.

14. Parietti V, Chifflot H, Muller S, Monneaux F. Regulatory T cells and systemic lupus erythematosus. Ann NY Acad Sci 2007; 1108: 64-75.

15. Alvarado-Sanchez B, Hernandez-Castro B, Portales-Perez $D$, et al. Regulatory T cells in patients with systemic lupus erythematosus. J Autoimmun 2006; 27: 110-8.

16. Valencia X, Yarboro C, Illei G, Lipsky PE. Deficient $\mathrm{CD} 4+\mathrm{CD} 25$ high $T$ regulatory cell function in patients with active systemic lupus erythematosus. J Immunol 2007; 178: 2579-88.

17. Crispin JC, Martinez A, Alcocer-Varela J. Quantification of regulatory $T$ cells in patients with systemic lupus erythematosus. J Autoimmun 2003; 21: 273-6.

18. Liu MF, Wang CR, Fung LL, Wu CR. Decreased CD4+CD25+ $T$ cells in peripheral blood of patients with systemic lupus erythematosus. Scand J Immunol 2004; 59: 198-202.

19. Suarez A, Lopez P, Gomez J, Gutierrez C. Enrichment of $\mathrm{CD} 4+\mathrm{CD} 25$ high $\mathrm{T}$ cell population in patients with systemic lupus erythematosus treated with glucocorticoids. J Ann Rheum Dis 2006; 65: 1512-7.

20. Zhang B, Zhang X, Tang FL, et al. Clinical significance of increased CD4+CD25-Foxp3+ T cells in patients with new-onset systemic lupus erythematosus. J Ann Rheum Dis 2008; 67: 1037-40.

21. Yan B, Liu Y. The nature of increased circulating CD4CD25Foxp3 T cells in patients with systemic lupus erythematosus: a novel hypothesis. Open Rheumatol J 2009; 3: 22-4.

22. Yang $H X$, Zhang $W$, Zhao LD, et al. Are CD4+CD25Foxp3+ cells in untreated new-onset lupus patients' regulatory T cells? J Arthritis Res Ther 2009; 11: R153.

23. Prado C, de Paz B, Lopez P, Gomez J, Rodriguez-Carrio J, Suarez A. Relationship between FOXP3 positive populations and cytokine production in systemic lupus erythematosus. J Cytokine 2012; 61: 90-6.

24. Suen JL, Li HT, Jong YJ, Chiang BL, Yen JH. Altered homeostasis of $\mathrm{CD} 4(+)$ FoxP3(+) regulatory T-cell subpopulations in systemic lupus erythematosus. I Immunol 2009; 127: 196-205.

25. Hochberg MC. Updating the American College of Rheumatology revised criteria for the classification of systemic lupus erythematosus. J Arthritis Rheum 1997; 40: 1725.

26. Warchol T, Lianeri M, Wudarski M, Lacki J, Jagodzinski P. Catalse $-262 C>T$ polymorphism in systemic lupus erythomatosus in Poland. J Rheumatol Int 2008; 28: 1035-9.

27. Long SA, Buckner JH. CD4+FOXP3+ T regulatory cells in human autoimmunity: more than a numbers game. J Immunol 2011; 187: 2061-6.

28. Suri-Payer E, Cantor H. Differential cytokine requirements for regulation of autoimmune gastritis and colitis by CD4(+)CD25(+) T cells. J Autoimmun 2001; 16: 115-23.

29. Bonelli M, Savitskaya A, von Dalwigk K, et al. Quantitative and qualitative deficiencies of regulatory $T$ cells in patients with systemic lupus erythematosus (SLE). Int Immunol 2008; 20: 861-8.

30. Bluestone JA, Tang Q, Sedwick CE. T regulatory cells in autoimmune diabetes: past challenges, future prospects. J Clin Immunol 2008; 28: 677-84.

31. Lin SC, Chen KH, Lin CH, Kuo CC, Ling QD, Chan CH. The quantitative analysis of peripheral blood FOXP3-expressing T cells in systemic lupus erythematosus and rheumatoid arthritis patients. Eur J Clin Invest 2007; 37: 987-96.

32. Yan B, Ye S, Chen G, Kuang M, Shen N, Chen S. Dysfunctional CD4+CD25+ regulatory T cells in untreated active systemic lupus erythematosus secondary to interferon-alpha-producing antigen-presenting cells. Arthritis Rheum 2008; 58: 801-12.

33. Shimizu J, Moriizumi E. Aging-dependent generation of suppressive CD4-CD25-R123lowCD103- T cells in mice. Eur J Immunol 2003; 33: 2449-58.

34. Shimizu J, Moriizumi E. CD4-CD25- T cells in aged mice are hyporesponsive and exhibit suppressive activity. J Immunol 2003; 170: 1675-82.

35. Nishioka T, Shimizu J, lida R, Yamazaki S, Sakaguchi S. CD4-CD25-Foxp3- T cells and CD4-CD25-Foxp3- T cells in aged mice. J Immunol 2006; 176: 6586-93.

36. Suen J, Chiang BL. CD4DFoxP3D regulatory T-cells in human systemic lupus erythematosus. J Formos Med Assoc 2012; 111: 465-70.

37. Tzeng TC, Suen JL, Chiang BL. Dendritic cells pulsed with apoptotic cells activate self-reactive T-cells of lupus mice both in vitro and in vivo. Rheumatology (Oxford) 2006; 45: 1230-7. 
38. Wan S, Xia C, Morel L. IL-6 produced by dendritic cells from lupus-prone mice inhibits CD4+CD25+ T cell regulatory functions. J Immunol 2007; 178: 271-9.

39. Bonelli M, Göschl L, Blüml S, et al. CD4+CD25-Foxp3+ T cells: a marker for lupus nephritis? J Arthritis Res Ther 2014; 16: R104.

40. Golding A, Hasni S, Illei G, Shevach EM. The percentage of FoxP3+Helios+ Treg cells correlates positively with disease activity in systemic lupus erythematosus. Arthritis Rheum 2013; 65: 2898-906.

41. Azab NA, Bassyouni IH, Emad Y, et al. CD4+CD25+ regulatory T cells (TREG) in systemic lupus erythematosus (SLE) patients: the possible influence of treatment with corticosteroids. J Clin Immunol 2008; 127: 151-7.

42. Lin SC, Chen KH, Lin CH, Kuo CC, Ling QD, Chan CH. The quantitative analysis of peripheral blood FOXP3-expressing $T$ cells in systemic lupus erythematosus and rheumatoid arthritis patients. Eur J Clin Invest 2007; 37: 987-96.

43. Lyssuk EY, Torgashina AV, Soloviev SK, Nassonov EL Bykovskaia SN. Reduced number and function of CD4+ CD25highFoxP3+ regulatory T cells in patients with systemic lupus erythematosus. J Adv Exp Med Biol 2007; 601: 113-9.

44. Vargas-Rojas MI, Crispin JC, Richaud-Patin Y, Alcocer-Varela J. Quantitative and qualitative normal regulatory $T$ cells are not capable of inducing suppression in SLE patients due to T-cell resistance. J Lupus 2008; 17: 289-94. 